\title{
The effect of delayed breast reconstruction after unilateral mastectomy on spine alignment
}

\author{
Joon Seok $\mathrm{Oh}^{1 \wedge}$, Hyoungmin $\mathrm{Kim}^{2} \wedge$, Ung Sik Jin ${ }^{1 \wedge}$ \\ ${ }^{1}$ Department of Plastic and Reconstructive Surgery, Seoul National University Hospital, Seoul National University College of Medicine, Seoul, \\ Korea; ${ }^{2}$ Department of Orthopedic Surgery, Seoul National University Hospital, Seoul National University College of Medicine, Seoul, Korea \\ Contributions: (I) Conception and design: US Jin, JS Oh; (II) Administrative support: US Jin; (III) Provision of study materials or patients: US Jin; \\ (IV) Collection and assembly of data: JS Oh, H Kim; (V) Data analysis and interpretation: JS Oh, H Kim; (VI) Manuscript writing: All authors; (VII) \\ Final approval of manuscript: All authors. \\ Correspondence to: Ung Sik Jin, MD, PhD. Professor, Department of Reconstructive Plastic Surgery, Seoul National University College of Medicine, \\ 101 Daehakro Chongno-gu, Seoul 03080, Korea. Email: usj1011@snu.ac.kr.
}

\begin{abstract}
Background Mastectomy in patients with breast cancer causes spinal deformities. We evaluated the effect of delayed breast reconstruction in post-mastectomy patients on spine alignments.

Methods: The study included 68 patients who underwent delayed breast reconstruction by three plastic surgeons in a single tertiary hospital. We measured proximal thoracic (PT), main thoracic (MT), and thoracolumbar (TL) Cobb angles and coronal spinal balance from chest or whole spine radiographs.

Results: The median changes in the PT and MT Cobb angles were -0.33 and -0.34 degrees, respectively. The change in TL Cobb angle and coronal spinal balance were only measured in 29 patients with available spine radiographs. The median change in TL Cobb angle and coronal spinal balance were -0.69 degrees and $3.75 \mathrm{~mm}$, respectively. The median preoperative and postoperative PT Cobb angles were 1.75 and 1.24 degrees. The difference between preoperative and postoperative PT Cobb angles was statistically insignificant $(\mathrm{P}=0.036)$. The median preoperative and postoperative MT Cobb angles were 1.32 and 1.09 degrees, respectively. The difference between preoperative and postoperative MT Cobb angles was statistically insignificant $(\mathrm{P}=0.221)$.

Conclusions: Delayed breast reconstruction did not result in clinically significant improvement in mastectomy-induced spinal deformity. This finding should be considered when choosing between immediate and delayed breast reconstruction.
\end{abstract}

Keywords: Breast cancer; delayed breast reconstruction; spine deformity; Cobb angles

Submitted Apr 18, 2021. Accepted for publication Jul 13, 2021.

doi: $10.21037 /$ gs-21-254

View this article at: https://dx.doi.org/10.21037/gs-21-254

\section{Introduction}

Breast cancer is the most common cancer in women (1). There were nearly 2 million newly diagnosed breast cancer patients in 2018 (2). Treatment options include mastectomy and breast conservative strategies. After treatment, patients may choose to live with an absent or deformed breast that results from mastectomy. Patients may also choose to undergo immediate or delayed breast reconstruction using prosthesis or autologous tissue transfer. The survival rate of breast cancer patients has risen recently (1). The 5 -year survival rate of women with invasive breast cancer is $91 \%$ and the 10 -year survival rate is $86 \%$ (3). Due to development in the detection and treatment of breast cancer, the survival rate has increased

^ ORCID: Joon Seok Oh, 0000-0002-8815-9555; Hyoungmin Kim, 0000-0002-4500-9653; Ung Sik Jin, 0000-0001-5096-6207. 
and the breast cancer mortality dropped by $40 \%$ from 1989 to 2007 (3). Along with improvements in survival rates of breast cancer patients, the rate of immediate and delayed breast reconstructions in the United States has increased over the last few decades (4). As a result, not only has there been extensive research about mastectomy and the overall curative process, but emphasis has also been placed on studies about postoperative quality of life of breast cancer surviving patients, especially those who undergo breast reconstruction. It is known that immediate breast reconstruction leads to improved self-esteem and emotional well-being (5-8).

One of the important aspects of breast reconstruction is the change in posture after mastectomy and breast reconstruction. Women's breasts play a significant role in upper body balance and posture. Lower back pain is commonly observed in many women after mastectomy $(6,9,10)$. Long-term spine deformity is observed in women undergoing unilateral mastectomy without reconstruction due to the imbalance caused by mastectomy (11-17). It has been shown that using an external prosthesis helped decrease the change in body posture caused by mastectomy (6). In addition, in one study, the magnitude of postoperative spinal deformity was smaller in patients who underwent immediate breast reconstruction compared to patients who underwent mastectomy without reconstruction (18). To our knowledge, there has not been a study to date that has evaluated the effect of delayed breast reconstruction on spinal deformity.

In this study, we analyzed the effect of delayed breast reconstruction on postoperative spinal deformity after total mastectomy in patients with breast cancer. We hypothesized that delayed breast reconstruction will not improve spinal deformity caused by mastectomy.

We present the following article in accordance with the STROBE reporting checklist (available at https://dx.doi. org/10.21037/gs-21-254).

\section{Methods}

This is a cohort study including 68 patients with a history of unilateral mastectomy, who underwent delayed breast reconstruction from August 2016 to October 2019 at the department of plastic and reconstructive surgery at a single tertiary hospital by three plastic surgeons. The study was conducted in accordance with the Declaration of Helsinki (as revised in 2013). The study was approved by Institutional Review Board (IRB No. 1606-056-770) and informed consent was taken from all the patients. For 31 patients, the study was conducted prospectively. The retrospective chart review included additional 37 patients.

A retrospective chart review was performed to collect patient information including age, height, weight, body mass index, type of reconstruction performed, implant/flap weight, date of mastectomy, and the period of delay before breast reconstruction.

Preoperative and post-operative whole spine or chest radiographs were used to analyze for spinal deformities. Whole spine radiographs were used for patients included in the prospective trial, and chest radiographs were used for patients analyzed retrospectively. Breast reconstruction was performed using free transverse rectus musculocutaneous (TRAM) flap, pedicled latissimus dorsi (LD) flap, and breast implant alone or combined with pedicled LD flap. All implant-based reconstructions were conducted in two-stages using a breast tissue expander. The flap inset weight and implant size were also recorded. Some patients also underwent contralateral reduction mammoplasty.

Multiple variables were measured to assess for spinal deformities. Cobb angles of proximal thoracic (PT), main thoracic (MT), and thoracolumbar (TL) were measured using preoperative and postoperative spine radiographs. For patients who only had chest radiographs and no spine radiographs, only the $\mathrm{PT}$ and $\mathrm{MT}$ Cobb angles were measured. PT Cobb angle is the angle formed between two parallel lines drawn from the superior end plate of the $\mathrm{T} 1$ vertebrae and inferior end plate of the $\mathrm{T} 3$ vertebrae. Similarly, MT Cobb angle is the angle formed between the superior end plate of the T3 vertebrae and inferior end plate of the T12 vertebrae. The TL Cobb angle is the angle formed between the superior end plate of the T12 vertebrae and inferior end plate of the L4 vertebrae. Each angle is shown in Figure 1.

In addition, coronal spinal balance was evaluated as shown in Figure 2 for patients who had whole spine radiographs. A vertical line was drawn from the middle point of the $\mathrm{C} 7$ vertebrae. Coronal spinal balance is defined as the horizontal distance between the drawn line and the midline of the coccyx. Coronal spinal balance is considered positive if the midline of the coccyx lies towards the patient's left side with respect to the vertical line by more than $2 \mathrm{~cm}$. It is considered negative if the midline lies towards the patient's right side with respect to the vertical line by more than $2 \mathrm{~cm}$. If the vertical line is within $2 \mathrm{~cm}$ away from the midline of the coccyx, the coronal spinal 

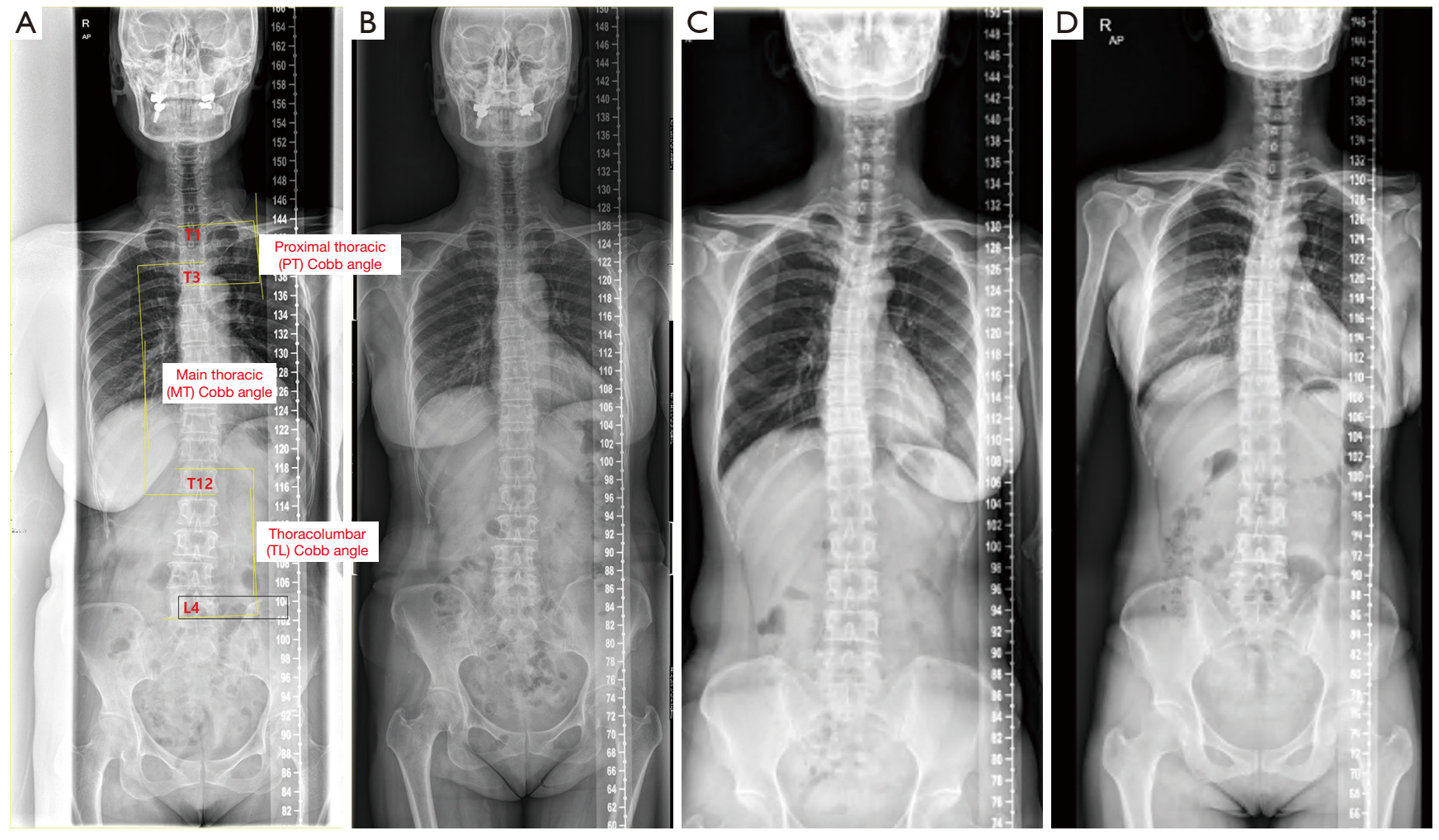

Figure 1 Evaluation of coronal Cobb angle (proximal thoracic main thoracic, thoracolumbar Cobb angle in (A) preoperative and (B) postoperative whole spine radiograph of a 57-year-old female patient with delayed breast reconstruction using free TRAM flap on her left breast with contralateral reduction mammoplasty on her right breast. (C) Preoperative and (D) postoperative whole spine radiograph of a 61-year-old female patient with delayed breast reconstruction using implant and pedicled LD flap on her right breast.

balance is considered neutral.

For patients who did not have whole spine radiographs taken and only chest radiographs were available, only the PT and MT Cobb angles were measured using the chest radiographs. A total of 29 patients had whole spine radiographs, while 39 patients had only chest radiographs.

The indications for extra $\mathrm{X}$-rays were unknown. No clinical information about the reasons for $\mathrm{X}$-rays were indicated in the electronic medical records. They were taken from other departments for unknown reasons.

For quality control, analysis of Cobb angle and coronal spinal balance were done by one plastic surgeon and one orthopedic surgeon who did not perform operation on any of the patients included.

\section{Statistical analysis}

All statistical analysis was performed by the Medical Research Collaborating Center Seoul National University
Hospital using SAS statistical software (SAS system for Windows, version 9.3; SAS institute, Cary, NC, USA). An independent $t$-test was performed to evaluate whether the postoperative changes in the Cobb angles and coronal spinal balance were statistically significant. A $t$-test or Wilcoxon rank sum test were performed to evaluate whether there was a difference in the distribution of coronal spinal balance, each Cobb angle, flap inset/implant weight and the patient's age. The Pearson correlation coefficient was evaluated. Both linear, and multiple regression analyses were performed to assess the relationship between each Cobb angle, flap inset/implant weight, patient age, height, weight, body mass index (BMI), duration between breast reconstruction and mastectomy, and duration between delayed breast reconstruction and exposure of the postoperative radiographs. Linear and multiple regression analysis were performed to assess the relationship between coronal spinal balances, flap inset/implant weight, patient age, and the duration without breast. 

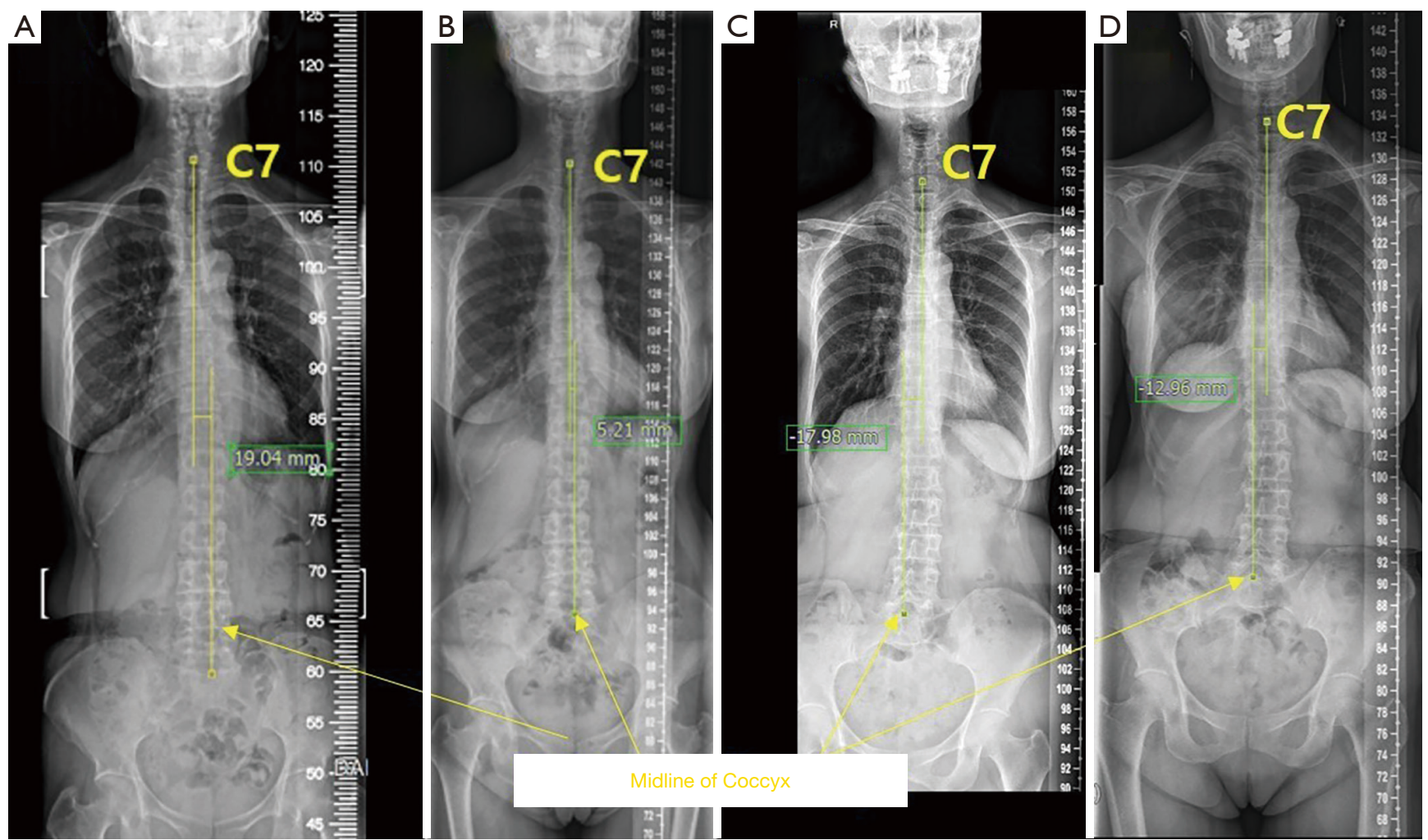

Figure 2 Evaluation of coronal spinal balance in (A) preoperative and (B) postoperative whole spine radiograph of a 57-year-old female patient with delayed breast reconstruction using free TRAM flap on her left breast with contralateral reduction mammoplasty on her right breast. (C) Preoperative and (D) postoperative whole spine radiograph of a 61-year-old female patient with delayed breast reconstruction using implant and pedicled LD flap on her right breast.

\section{Results}

A total of 68 patients were included in the study. Free TRAM flap was performed in 45 patients with 17 receiving contralateral reduction mammoplasty. Implant-based reconstruction was used in 23 patients, while 10 patients received a combined pedicled LD flap. Four patients received contralateral augmentation mammoplasty and one patient received contralateral reduction mammoplasty. Changes in the TL Cobb angle and coronal spinal balance were measured only in the 29 patients who had whole spine radiographs available pre- and postoperatively. For seven patients, the exact dates of mastectomy were unknown. The mass of mastectomy specimen is unknown for two reasons. Operative information regarding mastectomy was not available for patients who underwent mastectomy at different institutions. For patients who underwent mastectomy at our institution, mass of mastectomy specimen are measured only when immediate breast reconstruction is performed.

The preoperative and postoperative changes in the Cobb angles and coronal spinal balance are summarized in Table 1. The median of patient age, height, weight, BMI implant/flap inset weight, duration between mastectomy and delayed breast reconstruction, duration between delayed breast reconstruction and date of postoperative chest/spine radiograph, change in coronal spinal balance, and Cobb angle of PT, MT, and TL are listed in Table S1.

The median patient age was 50.07 years. The median height, body weight, and BMI of the patients were $158.35 \mathrm{~cm}, 59.1 \mathrm{~kg}$, and $23.65 \mathrm{~kg} / \mathrm{m}^{2}$, respectively. The median implant or flap weight was $450 \mathrm{cc}$ or gram. The median duration between mastectomy and delayed breast reconstruction was 6 years and seven patients were excluded from this calculation due to the exact date of mastectomy being unknown. The median duration between the date of breast reconstruction and postoperative radiograph was 2 years. The median changes in the PT and MT Cobb 
Table 1 Result of patient information and change in spinal factors of all 68 patients

\begin{tabular}{llc}
\hline Variable & $\mathrm{N}$ & Median (min max) \\
\hline Diff_PT (change in PT after operation) & 68 & $-0.33(-5.08,4.73)$ \\
Diff_MT (change in MT after operation) & 68 & $-0.34(-6.61,11.73)$ \\
Diff_TL (change in TL after operation) & 29 & $-0.69(-6.24,2.39)$ \\
Diff_Balance (change in coronal balance after operation) & 29 & $3.75(-21.81,26.71)$ \\
Type of breast reconstruction & & N/A \\
1: Free TRAM flap & 45 & N/A \\
2: Implant \pm pedicled LD flap & 23 & $450(210,995)$ \\
Implant/cc or flap inset mass $(g)$ & 68 & \\
\hline
\end{tabular}

Table 2 Preoperative and postoperative PT and MT Cobb angle measured from chest radiographs from 68 patients

\begin{tabular}{lcc}
\hline Variable & Median (min, max) & P value \\
\hline PT Cobb angle & & \\
Pre & $1.75(-3.64,15.37)$ & 0.036 \\
Post & $1.24(-2.68,11.72)$ & \\
MT Cobb angle & & \\
Pre & $1.32(-11.94,9.21)$ & 0.221 \\
Post & $1.09(-10.17,7.83)$ & \\
\hline
\end{tabular}

angles were -0.33 and -0.34 degrees, respectively. Changes in the TL Cobb angle and coronal spinal balance were only measured in 29 patients for whom whole spine radiographs were available. The median change in the TL Cobb angle and coronal spinal balance was -0.69 degrees and $3.75 \mathrm{~mm}$, respectively.

The preoperative and postoperative PT and MT Cobb angles are outlined in Table 2. The median preoperative and postoperative PT Cobb angles were 1.75 and 1.24 degrees, respectively. The mean difference between preoperative and postoperative PT Cobb angles were statistically insignificant $(\mathrm{P}=0.036)$. The median preoperative and postoperative MT Cobb angles were 1.32 and 1.09 degrees, respectively. The difference between preoperative and postoperative MT Cobb angles was statistically insignificant $(\mathrm{P}=0.221)$.

Linear regression analysis of the changes in PT and MT Cobb angles as the dependent variable are shown in Table 3 and Table 4. Compared to using free TRAM flap, the change in PT Cobb angle decreased by 0.26 degrees without statistical significance $(\mathrm{P}=0.645)$ for using implants with or without pedicled LD flap. Compared to using free TRAM flap, the change in MT Cobb angle increased by 0.344 degrees without statistical significance $(\mathrm{P}=0.670)$ for using implant with or without pedicled LD flap. Multiple linear regression analysis showed that changes in PT or MT Cobb angles were not dependent on any variables with statistical significance. The linear regression analysis results for changes in the TL Cobb angle and coronal spinal balance are shown in Table 5. Compared to free TRAM flap-based breast reconstruction, the TL Cobb angle of patients of implant with or without pedicled LD flapbased reconstructions increased by 0.8484 degrees without statistical significance $(\mathrm{P}=0.386)$. There was no statistically significant difference in postoperative changes in coronal spine balance or the TL Cobb angle between patients with free TRAM flap and those who had implant with or without pedicled LD flap-based reconstruction.

\section{Discussion}

Long-term spinal postural change is an important aspect that influences the postoperative quality of life in postmastectomy patients. Serel et al. (17) evaluated long-term spinal deformity by unilateral mastectomy in women. The postero-anterior chest radiographs of 60 women taken preoperatively and 1 year after mastectomy were analyzed. Spinal alignment generally tilted towards the contralateral side causing spinal deformity. The study concluded that the long-term spinal deformity may be caused by mastectomy due to the need to balance the absent ipsilateral breast

The effect of immediate breast reconstruction on preventing spinal postural changes has been observed in several studies. Ciesla and Polom (6) compared the 
Table 3 Linear regression of change in PT Cobb angle measured from chest radiographs from 68 patients

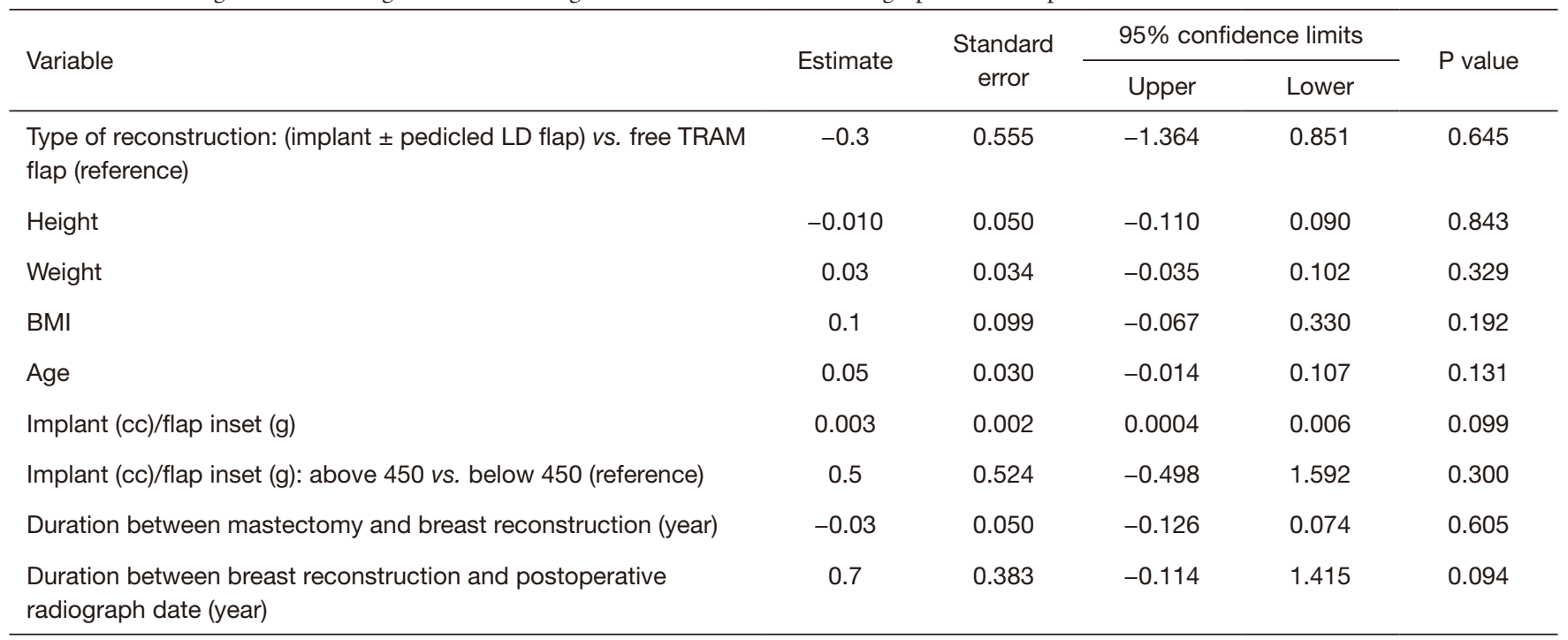

Table 4 Linear regression of change in MT Cobb angle measured from chest radiographs from 68 patients

\begin{tabular}{|c|c|c|c|c|c|}
\hline Variable & Estimate & $\begin{array}{l}\text { Standard } \\
\text { error }\end{array}$ & \multicolumn{2}{|c|}{$95 \%$ confidence limits } & $P$ value \\
\hline $\begin{array}{l}\text { Type of reconstruction: (implant } \pm \text { pedicled LD flap) } v s \text {. free } \\
\text { TRAM flap (reference) }\end{array}$ & 0.3 & 0.803 & -1.260 & 1.947 & 0.670 \\
\hline Height & 0.1 & 0.071 & -0.021 & 0.262 & 0.094 \\
\hline Age & -0.02 & 0.045 & -0.107 & 0.071 & 0.692 \\
\hline Implant (cc)/flap inset (g) & 0.001 & 0.002 & -0.004 & 0.005 & 0.738 \\
\hline $\begin{array}{l}\text { Implant (cc)/flap inset (g): above } 450 \text { vs. below } 450 \\
\text { (reference) }\end{array}$ & -0.5 & 0.761 & -2.061 & 0.977 & 0.479 \\
\hline $\begin{array}{l}\text { Duration between breast reconstruction and postoperative } \\
\text { radiograph date (year) }\end{array}$ & 0.4 & 0.564 & -0.701 & 1.550 & 0.454 \\
\hline
\end{tabular}

change in body posture among women who underwent radical mastectomy alone and those who underwent skinsparing mastectomy with immediate breast reconstruction using the Becker-25 prosthesis. Three-dimensional body surface analysis was performed in the coronal sagittal, and transverse planes preoperatively and 6, 12, 18 and 24 months postoperatively. Significant deviations from the proper body posture were observed in women who received radical mastectomy alone. The postural deviation first appeared 6 months postoperatively, and further deviations were observed until 18-24 postoperative months. In contrast, the changes in body posture among the women who received immediate reconstruction did not differ greatly from the control group.

Jeong et al. (18) retrospectively compared patients who received immediate breast reconstruction with mastectomy and patients who underwent mastectomy without breast reconstruction by analyzing Cobb angle 
Table 5 Linear regression of change in TL Cobb angle and coronal spinal balance from 29 patients who took preoperative and postoperative whole spine radiographs

\begin{tabular}{|c|c|c|c|c|c|c|}
\hline Variable & \multicolumn{3}{|c|}{ Change in TL Cobb angle } & \multicolumn{3}{|c|}{ Change in coronal spinal balance } \\
\hline \multicolumn{7}{|l|}{ Group } \\
\hline \multicolumn{7}{|l|}{ 1: Free TRAM flap (reference) } \\
\hline 2: Implant \pm pedicled LD flap & 0.84 & 0.9632 & 0.386 & -4.39 & 4.609 & 0.349 \\
\hline
\end{tabular}

between preoperative and 2-year postoperative chest radiographs. Significantly smaller changes in postoperative spinal deviation were observed in patients who underwent immediate breast reconstruction. This study showed the positive effect of immediate breast reconstruction in terms of spinal alignment.

Atanes Mendes Peres et al. (11) compared patients who underwent immediate breast reconstruction using an abdominal flap after mastectomy and patients who received mastectomy alone. Preoperative and postoperative trunk alignments were evaluated using the antero-posterior and lateral side views via the Postural Analysis Software/ Software de Análise Postural (PAS/SAPO). Significant differences in vertical trunk alignment were observed between the two groups. Greater trunk rotation was observed in the patients that did not undergo breast reconstruction. In contrast, no significant differences in head, shoulder, scapula or pelvis alignment were observed between the two groups.

In our study, the median change in the PT, MT, and TL Cobb angles were $-0.33,-0.34$ and -0.69 degrees, respectively. It is well recognized that a Cobb angle of $<10$ degrees is clinically insignificant (19). When spine deviates to the left or right, the condition is called scoliosis. Only when Cobb angles are greater than 10 degrees, spinal deviation is considered scoliosis. Cobb angles between 10 and 20 degrees are considered mild scoliosis. Cobb angles between 20 and 40 degrees are considered moderate scoliosis. Cobb angles greater than 40 degrees are considered severe scoliosis (19). Only three out of 68 patients had a preoperative Cobb angle $>10$ degrees. This means that most patients did not have a clinically relevant change in Cobb angle prior to breast reconstruction to induce scoliosis. The patients included in the study did not have a clinically relevant spinal deformity caused by mastectomy. The difference between preoperative and postoperative PT Cobb angles was statistically insignificant $(\mathrm{P}=0.036)$. However, the median preoperative, postoperative, and change in the $\mathrm{PT}$ Cobb angle were $1.75,1.24$ and -0.33 degrees which are substantially less than the 10 degrees cut-off. The median changes in other Cobb angles were also much smaller than 10 degrees. Thus, regardless of the preoperative spinal status of the patients, the breast reconstruction did not bring about a clinically relevant change in Cobb angle. Therefore, it is difficult to deduce that delayed breast reconstruction has a clinically relevant effect in spinal deformity.

Other factors such as age, height, body weight, BMI and implant or flap weight were not statistically associated with the change in Cobb angles. When reconstructing the breast, the goal is to match the size of the contralateral breast. Thus, patient height, body weight, BMI, and implant or flap weight may not correlate to the degrees of change in the Cobb angles.

All contralateral breast surgeries were done simultaneously with breast reconstruction. They were done only for cosmetic purposes. Regardless of whether contralateral mammoplasty was done, breast reconstruction was performed to match the contralateral breast in size. For this reason, we believe that whether the patient underwent contralateral breast surgery does not affect the study outcomes. Thus, this factor was not taken into account for evaluating the study outcome.

Coronal spinal balance is considered normal when the value is within $20 \mathrm{~mm}$. When the displacement is greater than $20 \mathrm{~mm}$, it is defined as coronal decompensation (20). The preoperative and postoperative coronal spinal balances of all patients were within $20 \mathrm{~mm}$. Thus, none of the patients showed coronal decompensation before undergoing breast reconstruction. There were some cases in which the displacement increased after breast reconstruction. This may be due to excessive compensatory spinal changes due to 
the breast reconstruction before finding a new stable spine position.

Serel et al. (17) showed that spinal deformity occurs after mastectomy even at postoperative year one. Jeong et al. (18) showed that spinal deformity occurs after mastectomy at postoperative year 2. In the present study, the average duration between breast reconstruction and mastectomy was 5 years. The longest period was 24 years and the shortest was 0.38 years. During the time delay, substantial changes in body posture may have occurred already. Moreover, this spinal deformity may be permanent after several years. Delayed breast reconstruction may not have a corrective effect on spinal alignment, since postural deviations have already occurred for a long time after mastectomy. A study with more patients who underwent delayed breast reconstruction within only a few years after mastectomy may be relevant in evaluating whether delayed breast reconstruction has a preventive effect on spinal deviations if performed early enough. The duration between breast reconstruction and mastectomy did not correlate with the changes in Cobb angles. As the average duration between breast reconstruction and mastectomy was 5 years, extra more years may not have influenced the outcome as the spinal deformity process must have completed within 5 years. Since most patients received breast reconstruction much later than two years, additional time lapse after the two years may not have led noticeable additional spinal deformity.

Postoperative radiographs were taken on average 1.48 years after the delayed breast reconstructions were performed. As such, there may not have been enough time for the spine to compensate and adjust, after implant or flap weight has been added to the affected side. If the postoperative Cobb angles and coronal spinal balance were measured at a much later time from the date of the breast reconstruction, the change in Cobb angles and coronal spinal balance may have been different.

\section{Limitation}

The study included only 68 patients. As the option of breast reconstruction immediately after mastectomy has increased, there are not plenty of patients who undergo delayed breast reconstruction these days to be included in the study (4). The study did not have information on the patient's spinal alignment before undergoing mastectomy. The study only evaluated the patient's spinal alignment just before delayed breast reconstruction and postoperatively. Therefore, there is no independent information regarding the magnitude of the effect of mastectomy alone on the patients' spinal alignment.

The study only evaluated patient's spinal posture in 2-dimensional radiographs although spine deformity can be more clearly evaluated in 3-dimensional imaging. As a result, it does not take into account any twists or torsional changes of the spine.

The study did not have control groups to compare the results with, for either women who did not undergo breast reconstruction or those who underwent immediate breast reconstruction. Women who underwent delayed rather than immediate breast reconstructions may be less concerned about their postoperative appearance, posture and quality of life in general. They may be less compliant and less motivated to engage in postoperative rehabilitation and maintaining a fit body. This may lead to worse spinal balance independent of mastectomy or breast reconstruction.

\section{Conclusions}

It is already known that mastectomy can negatively affect spinal posture and that immediate breast reconstruction can minimize this effect. In this study, preoperative and postoperative coronal spinal balance and the Cobb angles were measured in patients who underwent delayed breast reconstructions after unilateral mastectomies. In contrast to immediate breast reconstruction, it is inconclusive whether delayed breast reconstruction can prevent distortion of the spinal posture as the sample size of the study was not large enough to lead to any statistically significant conclusion. The coronal spinal balance tended to improve after delayed breast reconstruction, but was statistically insignificant. Although the study did not have a statistically significant conclusion, the result of this study still provides valuable information for both patients and surgeons in choosing reconstruction options for breast cancer patients, specifically in relation to the timing of reconstruction i.e., immediate $v s$. delayed breast reconstruction.

\section{Acknowledgments}

We acknowledge Medical Research Collaborating Center Seoul National University Hospital for performing all statistical analysis.

Funding: None. 


\section{Footnote}

Reporting Checklist: The authors have completed the STROBE reporting checklist. Available at https://dx.doi. org/10.21037/gs-21-254

Data Sharing Statement: Available at https://dx.doi. org/10.21037/gs-21-254

Conflicts of Interest: All authors have completed the ICMJE uniform disclosure form (available at https://dx.doi. org/10.21037/gs-21-254). The authors have no conflicts of interest to declare.

Ethical Statement: The authors are accountable for all aspects of the work in ensuring that questions related to the accuracy or integrity of any part of the work are appropriately investigated and resolved. The study was conducted in accordance with the Declaration of Helsinki (as revised in 2013). The study was approved by Institutional Review Board (IRB No. 1606-056-770) and informed consent was taken from all the patients.

Open Access Statement: This is an Open Access article distributed in accordance with the Creative Commons Attribution-NonCommercial-NoDerivs 4.0 International License (CC BY-NC-ND 4.0), which permits the noncommercial replication and distribution of the article with the strict provision that no changes or edits are made and the original work is properly cited (including links to both the formal publication through the relevant DOI and the license). See: https://creativecommons.org/licenses/by-nc-nd/4.0/.

\section{References}

1. Breast Cancer Statistics [database on the Internet] 2018. Available online: https://www.wcrf.org/dietandcancer/ cancer-trends/breast-cancer-statistics. Accessed: 202008-28.

2. Breast Cancer Statistics and Resources [database on the Internet]. Available online: https://www.bcrf.org/breastcancer-statistics-and-resources. Accessed: 2020-08-28

3. Breast Cancer: Statistics [database on the Internet] 2020. Available online: https://www.cancer.net/cancer-types/ breast-cancer/statistics. Accessed: 2020-08-28.

4. Panchal H, Matros E. Current Trends in Postmastectomy Breast Reconstruction. Plast Reconstr Surg 2017;140:7S-13S.
5. Al-Ghazal SK, Fallowfield L, Blamey RW. Comparison of psychological aspects and patient satisfaction following breast conserving surgery, simple mastectomy and breast reconstruction. Eur J Cancer 2000;36:1938-43.

6. Ciesla S, Polom K. The effect of immediate breast reconstruction with Becker-25 prosthesis on the preservation of proper body posture in patients after mastectomy. Eur J Surg Oncol 2010;36:625-31.

7. lder EE BY, Björklund T, Rylander R, et al. Quality of life and patient satisfaction in breast cancer patients after immediate breast reconstruction: a prospective study. Breast 2005;14:201-8.

8. Cordeiro PG, McCarthy CM. A single surgeon's 12year experience with tissue expander/implant breast reconstruction: part II. An analysis of long-term complications, aesthetic outcomes, and patient satisfaction. Plast Reconstr Surg 2006;118:832-9.

9. Rietman JS, Dijkstra PU, Hoekstra HJ, et al. Late morbidity after treatment of breast cancer in relation to daily activities and quality of life: a systematic review. Eur J Surg Oncol 2003;29:229-38.

10. Kopanski Z, Wojewoda T, Wojewoda A, et al. Influence of some anthropometric parameters on the risk of development of distal complications after mastectomy carried out because of breast carcinoma. Am J Hum Biol 2003;15:433-9.

11. Atanes Mendes Peres AC, Dias de Oliveira Latorre MD, Yugo Maesaka J, et al. Body Posture After Mastectomy: Comparison Between Immediate Breast Reconstruction Versus Mastectomy Alone. Physiother Res Int 2017;22.

12. Barbosa Jde A, Amorim MH, Zandonade E, et al. Evalution of body posture in women with breast cancer. Rev Bras Ginecol Obstet 2013;35:215-20.

13. Crosbie J, Kilbreath SL, Dylke E, et al. Effects of mastectomy on shoulder and spinal kinematics during bilateral upper-limb movement. Phys Ther 2010;90:679-92.

14. Hanuszkiewicz J, Malicka I, Stefańska M, et al. Body posture and trunk muscle activity in women following treatment of breast cancer. Ortop Traumatol Rehabil 2011;13:45-57.

15. Lapid O, de Groof EJ, Corion LU, et al. The effect of breast hypertrophy on patient posture. Arch Plast Surg 2013;40:559-63.

16. Rostkowska E BM, Samborski W. Body posture in women after mastectomy and its changes as a result of rehabilitation. Adv Med Sci 2006;51:287-97.

17. Serel S, Tuzlali ZY, Akkaya Z, et al. Physical Effects of Unilateral Mastectomy on Spine Deformity. Clin Breast 
Cancer 2017;17:29-33.

18. Jeong JH, Choi B, Chang SY, et al. The Effect of Immediate Breast Reconstruction on Thoracic Spine Alignment After Unilateral Mastectomy. Clin Breast Cancer 2018;18:214-9.

19. Horng MH, Kuok CP, Fu MJ, et al. Cobb Angle Measurement of Spine from X-Ray Images Using

Cite this article as: Oh JS, Kim H, Jin US. The effect of delayed breast reconstruction after unilateral mastectomy on spine alignment. Gland Surg 2021;10(8):2368-2377. doi: 10.21037/ gs-21-254
Convolutional Neural Network. Comput Math Methods Med 2019;2019:6357171.

20. Obeid I, Berjano P, Lamartina C, Chopin D, Boissière L, Bourghli A. Classification of coronal imbalance in adult scoliosis and spine deformity: a treatment-oriented guideline. Eur Spine J 2019;28:94-113. 
Supplementary

Table S1 Result of patient information and change in spinal factors

\begin{tabular}{|c|c|c|c|}
\hline Variable & $\mathrm{N}$ & Mean \pm S.D. & Median (Min Max) \\
\hline diff_MT (Change in MT after operation) & 68 & $-0.47 \pm 3.11$ & $-0.34(-6.61,11.73)$ \\
\hline diff_TL (Change in TL after operation) & 29 & $-0.98 \pm 2.09$ & $-0.69(-6.24,2.39)$ \\
\hline diff_Balance (Change in coronal balance after operation) & 29 & $2.74 \pm 10.04$ & $3.75(-21.81,26.71]$ \\
\hline - 1: free TRAM flap & 45 & $\mathrm{~N} / \mathrm{A}$ & $\mathrm{N} / \mathrm{A}$ \\
\hline - 2: Implant \pm pedicled LD flap & 23 & $\mathrm{~N} / \mathrm{A}$ & $\mathrm{N} / \mathrm{A}$ \\
\hline Height/cm & 68 & $158.05 \pm 5.3$ & $158.35(144.6,171.3)$ \\
\hline Body weight $/ \mathrm{kg}$ & 68 & $59.31 \pm 7.67$ & $59.3(43.5,82.65)$ \\
\hline Impant/cc or Flap inset mass(g) & 68 & $486.01 \pm 169.25$ & $450(210,995)$ \\
\hline \multicolumn{4}{|l|}{ Impant(cc)/Flap inset(g) } \\
\hline - Below 400 & 31 & $\mathrm{~N} / \mathrm{A}$ & N/A \\
\hline - Above 400 & 37 & $\mathrm{~N} / \mathrm{A}$ & $\mathrm{N} / \mathrm{A}$ \\
\hline $\begin{array}{l}\text { Duration between mastectomy and delayed breast } \\
\text { reconstruction/ year }\end{array}$ & 62 & $5.02 \pm 5.71$ & $3.05(0.38,24.04)$ \\
\hline $\begin{array}{l}\text { Duration between breast reconstruction and postoperative } \\
\text { radiograph /year }\end{array}$ & 68 & $1.48 \pm 0.68$ & $1.34(0.32,3.18)$ \\
\hline
\end{tabular}

